\title{
Core X-ray sources in the Local Group galaxies
}

\author{
E. J. A. Meurs \\ Dunsink Observatory, Dublin Institute for Advanced Studies, Castleknock, Dublin 15, IRL \\ email: ejam@dunsink.dias.ie
}

\begin{abstract}
As the nearest galaxies around us, the Local Group systems offer especially good opportunities for observations of their nuclear X-ray radiation. Certain or possible nuclear X-ray sources in the Local Group suggest a minimum luminosity for activity to become manifest.
\end{abstract}

Keywords. galaxies: active galactic nuclei, Local Group, X-rays: galaxies.

\section{Introduction: prevalence of active cores in galaxies}

Among the many individual X-ray sources that nowadays are seen in neighbouring galaxies, the nuclear cores take a special position. Not only do they tend to be the centralmost source, but all other individual sources are products of stellar processes while the nuclear cores constitute the (usually) one and only source in a galaxy that is instead related to the supermassive black holes that power QSOs on a cosmic scale. There may incidentally be a kind of intermediate class of sources (that is, between stellar source and core source), the UltraLuminous X-ray sources that have also been discussed extensively during this Symposium, but their precise nature is still not conclusively established. There is still another type of source, which is mostly the result of stellar processes, diffuse X-ray emission, but we will not consider such emission here, as not being individual sources.

Nearby galaxies are usually no QSOs, fortunately enough, and from the nearest galaxies to the luminous QSOs far away spans a large range in level of nuclear activity. Upon closer examination, some form of nuclear activity can often be recognized in the centres of many galaxies around us. It is usually thought that remnant supermassive black holes, left behind at a period of higher nuclear activity some time in the past, make their presence known in these instances, when they receive fuel in the form of gas or stars.

The recognition of weak forms of nuclear activity leads naturally to questions like: how frequent is nuclear activity present among galaxies; do all types of galaxies exhibit nuclear activity; is a minimum size (i.e., mass) required for galaxies in order to harbour a nuclear core; till what low level of energy output can active cores be recognized. Obviously, the closer the galaxies, the easier to address these questions. Therefore, in this contribution, we restrict ourselves to the very nearest galaxies, those of the Local Group. Their small distances yield the best spatial resolution, notably important when low flux levels are reached at which cores would be comparable to all kinds of stellar objects.

Using X-rays is efficient in the search for low-level cores. Nuclear cores emit notably at high energies, as is known from e.g. Seyfert and Liner galaxies. Other objects in the galaxies are generally less likely to emit at X-rays, thus compared with all stellar objects in a galaxy there are less competitors for our attention. 


\section{The Local Group sample}

The Local Group (LG) contains at least some 36 galaxies, from the big spirals (M31; our own Galaxy) down to small and loose aggregations of stars (e.g. the Draco dwarf galaxy). The number of LG members may vary from author to author. Important for our purposes is that galaxies of nearly all sorts are present: spirals (our Galaxy; M31; M33), ellipticals (e.g. NGC147 and NGC185) and irregulars (e.g. NGC6822); but no lenticular. Also, within these classes they show a range of sizes, although notably there is no normally sized elliptical. Below we will exclude the two Magellanic Clouds, which are very extended objects without clear centres in their stellar distribution.

\section{Existing X-ray surveys of the Local Group}

\section{(a) Einstein}

Results were obtained for about six LG members, this work has been described by Helfand (1984) and Fabbiano (1989). The presence of a strong central source in M33 was noted.

\section{(b) ROSAT}

A good number of the LG galaxies was observed with ROSAT. This was the basis for an X-ray survey of their cores (Zang \& Meurs 2001; Meurs 2003).

\section{(c) Chandra and XMM-Newton}

The two currently flying X-ray satellites have so far yielded results for some of the LG galaxies, and several more are in the process of being observed, being analysed, or being published.

In the sense of providing an assessment of the cores of LG galaxies, the most comprehensive overview to date is by Zang \& Meurs (2001), employing the ROSAT database. For some of these galaxies, Chandra and/or XMM-Newton data improve on the ROSAT results. In the next Section, therefore, we review the currently available X-ray data on LG galaxy cores. In the Section following after that, a general discussion of the LG cores is given, based on Zang \& Meurs with updates where relevant.

\section{Cores of individual Local Group galaxies}

\section{(i) Galactic Centre}

Using the ROSAT PSPC, Predehl \& Trümper (1994) found a source coincident with Sgr $\mathrm{A}^{*}$ within $10 \operatorname{arcsec}\left(\Delta_{X O}=7.8 \operatorname{arcsec}\right)$, visible only above $1.2 \mathrm{keV}$. With the greater spatial resolution of Chandra, Baganoff et al. (2003) resolve the source seen with ROSAT into a number of components, the strongest of which is only 0.27 arcsec from the radio position as determined interferometrically by Reid et al. (1999). The flux in the 0.1$2.4 \mathrm{keV}$ band is about two orders of magnitude less than was found with ROSAT.

A flare of limited duration and magnitude may have occurred, but this does not seem to be a very regular phenomenon (see also Baganoff et al. 2001). Insights on the activity history of the Galactic Centre are found in Koyama (this volume).

\section{(ii) $M 31$}

One of the sources discerned with the ROSAT HRI was coincident with the nucleus of M31 (Primini et al. 1993). This source could be resolved into 4 or 5 sources by Chandra (Garcia et al. 2000). Chandra and HST images have subsequently been registered to an accuracy of 0.1 arcsec (Garcia et al. 2005), resulting in a $2.5 \sigma$ detection of a source directly next (West) to the northernmost of the nuclear Chandra sources in Garcia et al. (2000). The coincidence with the nuclear radio source (Crane et al. 1992) is within 1 arcsec, the X-ray luminosity in $0.1-2.4 \mathrm{keV}$ is down to $\log \mathrm{L}_{X}=35.93$. 
Table 1. Core sources in LG galaxies

\begin{tabular}{lccr}
\hline Galaxy & $\begin{array}{c}\log \mathrm{L}_{X} \\
(0.1-2.4 \mathrm{keV})\end{array}$ & $\begin{array}{c}\Delta_{X O} \\
(\operatorname{arcsec})\end{array}$ & $\begin{array}{r}\mathrm{M}_{S M B H} \\
\left(\mathrm{M}_{\odot}\right)\end{array}$ \\
\hline M33 & 39.01 & $<1$ & $<1.510^{3}$ \\
M32 & 36.45 & 0.7 & $2.510^{6}$ \\
M31 & 35.93 & $<1$ & $310^{7}$ \\
GC & 34.66 & 0.27 & $2.610^{6}$ \\
\hline
\end{tabular}

(iii) $M 33$

The bright X-ray source in the nucleus of M33 has been known since the Einstein observations (e.g. Fabbiano 1989). It is the most luminous source in the LG (M33 X-8) and qualifies as an UltraLuminous X-ray source. Analysis of ROSAT observations led to a suggested long binary period (Dubus et al. 1997), which in later work could not be confirmed (Parmar et al. 2001, using BeppoSAX). The currently most popular explanation probably is a microquasar-like X-ray binary (Dubus \& Rutledge 2002), supporting evidence has been derived from spectral analyses and short term variability at X-rays (La Parola et al. 2003; Foschini et al. 2004; Dubus et al. 2004). The close coincidence of M33 X-8 with the nucleus of M33, to within 0.6 arcsec (Dubus \& Rutledge 2002), is nevertheless very noticeable (Schulman \& Bregman 1995). A putative central black hole may have a mass in such an uncommon range $\left(<1500 \mathrm{M}_{\odot}\right.$, Gebhardt et al. 2001$)$ that spectral models pursued so far are not entirely appropriate. Therefore we include, for the time being, the M33 central source as a possible nuclear core, as in Zang \& Meurs (2001).

(iv) $M 32$

Based on analysis of ROSAT PSPC and HRI observations, Zang \& Meurs (1999) advanced reasons that the bright central source in this small elliptical galaxy could conceivably be a low level active nucleus. Using Chandra data, Ho et al. (2003) report a weak source at 0.7 arcsec from the 2MASS position of M32. Adopting the latter result, the luminosity in the $0.1-2.4 \mathrm{keV}$ band is $\log \mathrm{L}_{X}=36.45$.

(v) NGC6822

Zang \& Meurs (2001) found an intriguing, weak source in a ROSAT HRI observation, only $\sim 7$ arcsec away from the nominal optical position of the galaxy. NGC6822 is however an irregular galaxy, without well-defined centre, thus it is difficult to attribute this source to a core. We glanced at a later Chandra observation of this galaxy (J. Hartwell, private communication), but this has not very good statistics.

(vi) $W L M$

This is also an irregular galaxy, posing the same problems as for NGC6822. ROSAT HRI data indicate a source only $\sim 8$ arcsec from the nominal optical position (Zang \& Meurs 2001).

Thus, X-ray detection of core sources in LG galaxies include the Galactic Centre, M31 and M32, and possibly M33. The central sources in NGC6822 and WLM require more extensive observational studies than available to date.

\section{Discussion: core X-ray sources in the Local Group}

The recent advances in high resolution X-ray imaging of LG galaxies as quoted in the previous Section leave the main conclusions of Zang \& Meurs (2001) unaffected. The 
current status of core X-ray sources in the LG is summarized in Table 1; the last column gives the estimated mass of a central supermassive black hole.

The most important results on galaxy cores in the LG are:

(1) $\mathrm{Sgr} \mathrm{A}^{*}$ in the Galactic Centre is still the weakest detected nuclear core within the LG.

(2) The nucleus of M33 remains the strongest nuclear source within the LG - if indeed it is one.

(3) All three spiral galaxies in the LG have a nuclear X-ray source (when including M33).

(4) Only the most luminous (dwarf) elliptical, M32, has a nuclear source.

(5) None of the irregular galaxies features a recognizable core source.

(6) Galaxies seem to need $\mathrm{L}_{B}>10^{8} \mathrm{~L}_{B, \odot}$ for having a core source.

The LG conclusions are based on X-ray observation of 25 out of (ca.) 36 galaxies. Apart from M33, the detected core sources exhibit luminosities as for stellar X-ray sources. It will be interesting to obtain similarly good coverage (as to number of members observed) for next following nearby groups. The Chandra survey of nearby galaxies by Ho et al. (2001) emphasizes AGN candidates. Another way to recognize non-AGN cores, albeit very incidentally, is to observe stellar disruption flares that temporarily highlight otherwise dormant supermassive black holes in galaxy centres (e.g. Cunniffe 2003).

\section{References}

Baganoff, F.K., Maeda, Y., Morris, M., et al. 2003, ApJ 591, 891

Crane, P.C., Dickel, J.R., \& Cowan, J.J. 1992, ApJ 390, L9

Cunniffe, J. 2003, PhD Thesis, Trinity College Dublin, Ireland

Dubus, G., et al. 1997, ApJ 490, L47

Dubus, G. \& Rutledge, R.E. 2002, MN 336, 901

Dubus, G., Charles, P.A., \& Long, K.S. 2004, A\&A 425, 95

Fabbiano, G. 1989, ARAA 27, 87

Foschini, L., Rodriguez, J., Fuchs, Y., et al. 2004, A\&A 416, 529

Garcia, M.R., Murray, S.S., Primini, F.A., et al. 2000, ApJ 537, L23

Garcia, M.R., Williams, B.F., Yuan, F., et al. 2005, ApJ 632, 1042

Gebhardt, K., Lauer, T.L., Kormendy, J., et al. 2001, AJ 122, 2469

Helfand, D. 1984, PASP 96, 913

Ho, L.C., Feigelson, E.D., Townsley, L.K., et al. 2001, ApJ 549, L51

Ho, L.C., Terashima, Y., \& Ulvestad, J.S. 2003, ApJ 589, 783

La Parola, V., et al. 2003, ApJ 583, 758

Meurs, E.J.A. 2003, A\&A 408, 95

Parmar, A.N., Sidoli, L., Oosterbroek, T., et al. 2001, A\&A 368, 420

Predehl, P. \& Trümper, J. 1994, A\&A 290, L29

Primini, F.A., Forman, W., \& Jones, C. 1993, ApJ 410, 615

Reid, M.J., et al. 1999, ApJ 524, 816

Schulman, E. \& Bregman, J.N. 1995, ApJ 441, 568

Zang, Z. \& Meurs, E.J.A. 1999, NewA 4, 521

Zang, Z. \& Meurs, E.J.A. 2001, ApJ 556, 24

\section{Discussion}

LIPUNOV: I remember that before the Chandra era there was some excess of the $\mathrm{BH}$ candidate systems in the central part of our Galaxy. What is the situation now?

Meurs: The number of candidate supermassive black holes in the Galactic Centre is essentially down to one candidate, for which there are indications at a variety of wavelengths. 